\title{
Click::RAND. A Minimalist Sound Sculpture
}

\author{
Paul Dunham \\ Victoria University of \\ Wellington \\ PO Box 600 \\ Wellington 6140 \\ New Zealand \\ paul.dunham@vuw.ac.nz
}

\author{
Mo. H. Zareei \\ Victoria University of \\ Wellington \\ PO Box 600 \\ Wellington 6140 \\ New Zealand \\ mo.zareei@vuw.ac.nz
}

\author{
Dugal McKinnon \\ Victoria University of \\ Wellington \\ PO Box 600 \\ Wellington 6140 \\ New Zealand \\ dugal.mckinnon@vuw.ac.nz
}

\author{
Dale Carnegie \\ Victoria University of \\ Wellington \\ PO Box 600 \\ Wellington 6140 \\ New Zealand \\ dale.carnege@vuw.ac.nz
}

\begin{abstract}
Discovering outmoded or obsolete technologies and appropriating them in creative practice can uncover new relationships between those technologies. Using a media archaeological research approach, this paper presents the electromechanical relay and a book of random numbers as related forms of obsolete media. Situated within the context of electromechanical sound art, the work uses a non-deterministic approach to explore the non-linear and unpredictable agency and materiality of the objects in the work. Developed by the first author, Click::RAND is an object-based sound installation. The work has been developed as an audiovisual representation of a genealogy of connections between these two forms of media in the history of computing.
\end{abstract}

\section{Author Keywords}

media archaeology, object-based, sound installation, electromechanical

\section{CCS Concepts}

- Applied computing $\rightarrow$ Arts and humanities $\rightarrow$ Media arts • Applied computing $\rightarrow$ Arts and humanities $\rightarrow$ Sound and music computing $\bullet$ Hardware $\rightarrow$ Communication hardware, interfaces and storage $\rightarrow$ Electro-mechanical devices

\section{INTRODUCTION}

In his (an)archaeological quest to research the deep time of media history, Siegfried Zielinski suggests we need to adopt an unfamiliar perspective to find something new in the old [1]. As such, media archaeology is one approach to re-presenting obsolete media in new contexts and changing our perspective of and/or relationship with the object. By presenting obsolete objects in new or unfamiliar contexts in creative practice, new perspectives of the object and its relationship to other media can emerge. Exploring, manipulating, and foregrounding the use of appropriated and outmoded objects for the purpose of creating sound-based installations and performances is one way this can be achieved.

This paper presents an object-based sound installation that, through a media archaeological approach, connects a well-known mid-20th

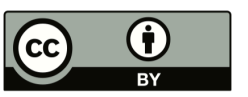

Licensed under a Creative Commons Attribution 4.0 International License (CC BY 4.0). Copyright remains with the author(s).

NIME'20, July 21-25, 2020, Royal Birmingham Conservatoire, Birmingham City University, Birmingham, United Kingdom.

\footnotetext{
${ }^{1}$ A relay is an electro-mechanical switch that controls one circuit by opening and closing another.
}

century book of random numbers with the electro-mechanical relay ${ }^{1}$ as historically related media artefacts. Titled Click::RAND, the work foregrounds the sound-producing object by visibly connecting the sound to the sound source and uses the sonic by-products of a series of relays to create a sound installation. The relays are actuated based on the random selection of numbers extracted from The RAND Corporation's book, A Million Random Digits with 100,000 Normal Deviates [2]. Click::RAND utilises a series of relays and LED lights to provide an audio-visual representation of the book. Through its performative presence, the work creates a relationship between early electro-mechanical computers which used relays as a constructive part of their mechanism and random number sets generated by other means. As such, Click::RAND is an audio-visual representation of distinctly different, but genealogically related, forms of media.

The next section provides a brief background review of related work. Section 3 introduces Click::RAND, outlining various technical and compositional strategies employed in its development. This is followed by a brief discussion on future works, before ending with concluding remarks.

\section{BACKGROUND}

The use of probabilistic methods and pseudo-random number generators is common across a wide range of practices within experimental music and sound art. The use of stochastic methods of composing or performing music has a long history. Some of the descriptions for these techniques include, but are not limited to, aleatoric, non-deterministic or chance music. More recent precedents for the use of these forms include Marcel Duchamp's Erratum Musical (1913), John Cage's Music of Changes (1951), Iannis Xenakis' Pithoprakta (1956) and performances of Karlheinz Stockhausen's Klavierstück XI (1956). Today, these techniques are used to create a lack of pattern or predictability by a large number of artists.

The use of a non-deterministic approach in electromechanical sound art allows an artist to explore the agency and behaviour of materials and objects in their works. Jon Pigott describes this as a process-driven sensibility that exhibits unpredictable sounding, non-linear and unpredictable material behaviours [3]. He argues that, as a creative method, the artist relinquishes an element of control by letting the creative process play a part in the natural unfolding of events. Examples of the use of unpredictability in the resultant sound work include Stephen Cornford's Migration (2014), Darsha Hewitt's Electrostatic Bell Choir (2012-2013), a number of Sergey Filatov's sound sculptures and many of Zimoun's works.

Utilising random numbers to actuate a series of electromechanical relays, Click::RAND shares a connection with other 
similar sound-based works that use a non-deterministic approach to explore the material behaviours of objects. Engaging with the sound producing properties of the relay, the following section introduces Click::RAND, an audio-visual representation of the media archaeological connection between the relay as a key element in early computers and a book of random numbers.

\section{Click::RAND}

Developed by the first author, Click::RAND ${ }^{2}$ was conceptualised as a sonic articulation of The RAND Corporation's book $A$ Million Random Digits with 100,000 Normal Deviates (see Figure 1).

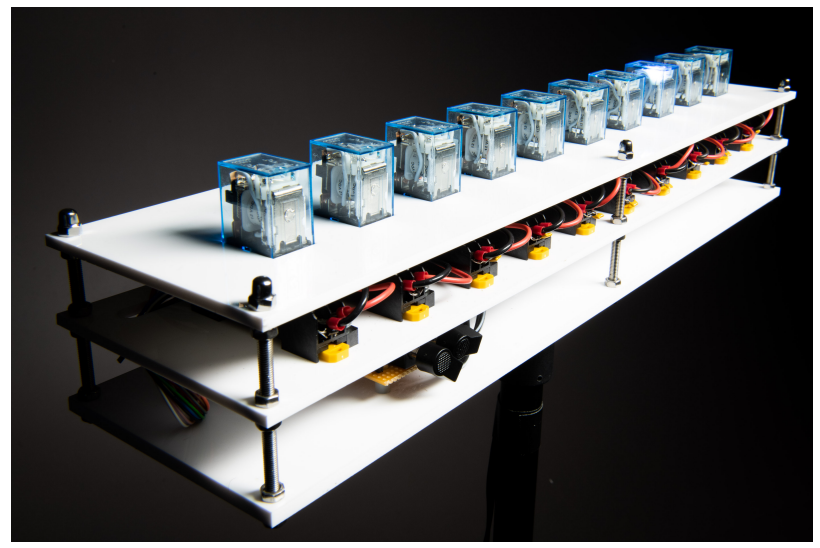

Figure 1. Click::RAND

\subsection{Background}

A book noting the unreliability of early relay-based computers formed the basis of considering how the material sound of the relays could be used to create a sound-based installation [4]. The failure of those relays not only caused computational failure but also disrupted the patterns and rhythms associated with the computer's electro-mechanical elements. Subsequent research highlighted the fact that early computers were also not capable of generating large-scale true random number sets, a process that still challenges modern computing systems. Researchers and scientists at the time turned to other methods to create those sets. A Million Random Digits with 100,000 Normal Deviates was published in 1955 to supply a large set of random numbers to help solve various problems by "experimental probability procedures" in diverse areas including research, statistics, physics, poll taking, market analysis, scientific experiment design and cryptography [2]. The random number and normal deviation data sets are now freely available from The RAND Corporation's website ${ }^{3}$. Could this data be transformed and utilised to create a connection between the book and the electromechanical computer? Could the electromechanical relay be appropriated, and its sonic by-products be used to create a sound-based work?

Click::RAND is an effort to explore these questions. The work utilises the sound produced by a single relay as the entire raw material. Such a reductionist approach is employed to highlight the relay's sonic characteristics. This sound is heard as a double click when the relays contact points engage and disengage. As such, the compositional palette is very limited. Click::RAND's use of ten relays as an iteration of the single unit is a way of expanding the sonic palette and of exposing the slight nuances of each relay.

\footnotetext{
2 A short video of Click::RAND is available at https://tinyurl.com/v8levx5
}

\subsection{Overview}

Click::RAND uses relays as an historical connection to their use in early mechanical and relay computers (e.g., Konrad Zuse's Z2, Howard Aiken's MARK, Edward Condon's Nimatron). A series of ten relays represent digits from 0 to 9 which, in turn, represent each random number from the book. As each random number is played, the representative relay clicks and an embedded LED flashes (see Figure 2). It has been argued that the presence of a visual cue in relation to the sound can be an access tool for increasing audience engagement with a work [5]. The LED reinforces the sonic experience by providing a visual connection to the sound source. Unless the audience is close to the work, it can be difficult to determine the individual source from sound alone. In this way the audience can connect the sonic nuances to a specific sound object.

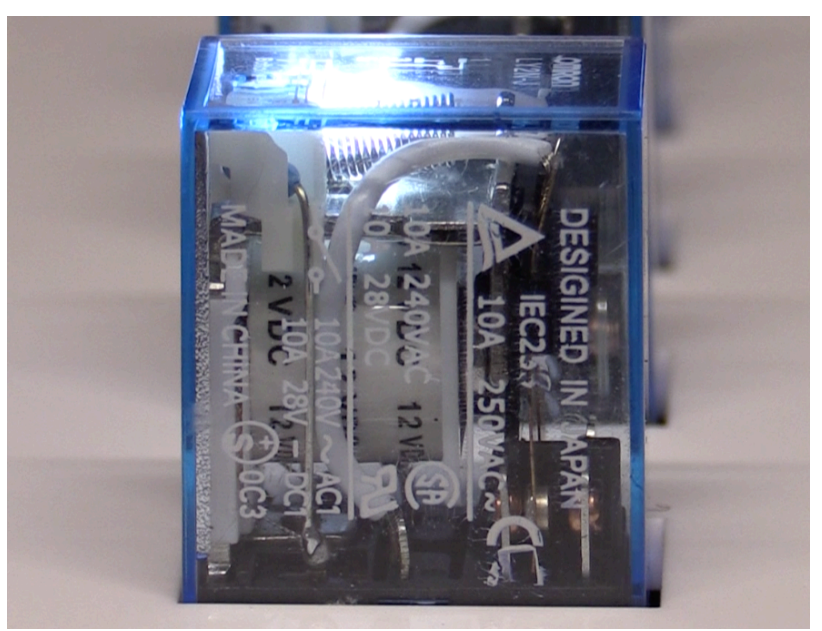

Figure 2. Single Relay with LED

The action of each relay is powered through and programmatically controlled by a Teensy microcontroller via a MOSFET driver circuit. A series of files containing the data set are stored on the Teensy's memory card. The Teensy is programmed to randomly select a file from those stored on a memory card. The data contained within the file is then stored in a data array. A number is randomly selected from the array used to actuate the associated relay. A high-level system overview is given in Figure 3.

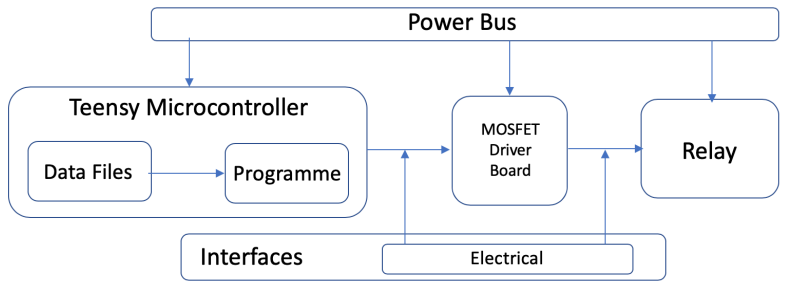

Figure 3 Click::RAND high-level system overview

Click::RAND is presented with the original data source, the book, as an explicit part of the work (see Figure 4). As such, the interpretation of the book's content and subsequent representation in the audio-visual domain has created a new perspective for interpretation.

Click::RAND's sonic material is provided by relay contacts, each heard as a click when they engage and disengage. As such,

${ }^{3}$ https://www.rand.org/pubs/monograph_reports/MR1418.html 
the sonic palette appears to be limited to that series of clicks. However, differences can be heard in the sound of the contacts of some relays when they are actuated. Some are muted, some have a resonant ring to them, some are simply a metallic click. For some relays, other elements of their material construction can be heard as, what might be named, micro-sounds. These micro-sounds are not a glitch but are a part of the inherent material characteristics of the relay. As such these acoustic micro-sounds become a part of the work and are integrated into its overall sonic palette.

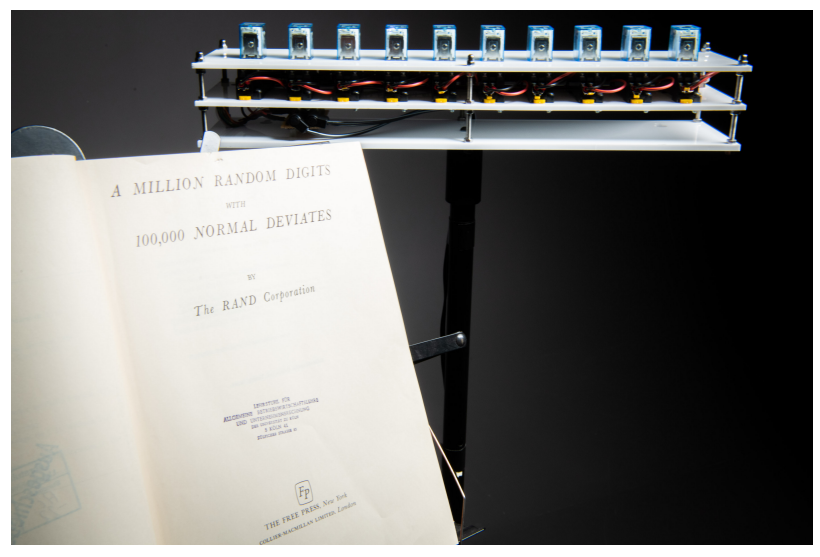

Figure 4. Click::RANDs physical structure

\subsection{Compositional Strategies}

The book's instructions for use of the tables (see below) has been the primary source for informing a compositional strategy for Click::RAND.

In any use of the table, one should first find a random starting position. A common procedure for doing this is to open the book to an unselected page of the digit table and blindly choose a five-digit number. [2]

This instruction has been liberally interpreted and incorporated into the software that controls the work. In this case, a single digit is selected. This random selection to actuate the relays can be perceived as selecting a new starting point each time by opening the book to an unselected page and choosing a number.

Incorporating musical elements when using non-musical sounds is a strategy to help engage and hold audience attention [6] [7]. However, Scott Gresham-Lancaster contends that real interest can lie in the "discovery of unpredictable and emergent sound qualities" resulting from abstract interrelationships between the source data and the sonic output of a given process or set of processes [8]. Adopting this latter approach, Click: $: R A N D$ eschews the use of musical elements such as a regular tempo or rhythm. Instead, by using the normal deviate data set as a temporal element, it is left to random processes for patterns to briefly emerge and ebb. This approach allows a listener's focus to shift between two levels. At the macro-level are the emergent and ebbing patterns as a part of the work as a whole. At a micro-level the randomness that disrupts those patterns draws attention to the individual sounds. As such, a listener's attention is always in flux as it shifts between these levels; this may not be the case if the use of a regular tempo or rhythm was employed. Finally, by using the normal deviate numbers, those irregular time intervals between relay actuations are analogous to the disrupted patterns and rhythms heard in the functioning of the original electromechanical computers.

\section{Extending Click::RAND}

Click::RAND has been presented in a number of diverse venues. These have ranged from an intimate space for the work and a small audience, a multi-purpose presentation room, and public spaces including foyers and event centres. In its current form, Click::RAND has a small presence both physically and sonically. The work responded best in the more intimate space. In this environment, the subtle sounds can be best heard; the work encouraged the audience to get close to hear and see its characteristics nuances. In a competing space, the ability to experience the work closely was diminished and the subtle sounds were lost in the milieu.

Reflecting on Click::RAND, the work's physical form and ways of interpreting and utilising the random number set have the opportunity to be explored and extended. The current work is informed by the random number range in the book. As such, the work is presented as a linear form, counting 0-9, left to right in a somewhat conventional form.

Click::RAND can be extended to create a larger and more immersive presence. By creating a series of modular 'sound blocks' that iterate the basic sound unit (the relay), the work can be adapted for a range of spaces and for the data to be used in ways that explore and expose the rhythmic qualities of the data patterns? Adapting these 'sound blocks' to the space the work inhabits can be a way of exploring the different acoustic quality of different spaces that create a different sonic interpretation of the work for each installation. Combining this physical approach with different interpretations of the data can be a way of creating new spatialised sound patterns such as syncopated rhythms. Spatialising the work also offers an opportunity to experience it in different ways as listeners move through the space. This approach may result in a more complex sensory exploration which is representative of the complexity of A Million Random Digits with 100,000 Normal Deviates.

Iterating the basic sound unit as a series of 'sound blocks' can be one way of interpreting the physical layout of the numbers in the book as the basis for presenting the work. Adapting the layout of the work in this way would also strengthen the connection between the work and the presence of the book. By iterating the basic sound unit across a number of modules, an audio-visual presence can be created with its physical layout and allow variations in the density and spatialisation of sound. The reconfigurable physical structure of the work will allow a number of compositional approaches to be explored. One such approach can be to explore the numbers as a series of binary sequences allowing other rhythmic forms and patterns to be presented. Again, by iterating the single unit via a series of 'sound blocks', Click::RAND can be extended to create a multi-dimensional and adaptable series of works to explore bot the inherent rhythmic patterns in the data and the acoustic nuances of the space it inhabits.

\section{Conclusion}

Following outmoded or obsolete media can uncover a genealogy between media that may not be immediately obvious. Creatively appropriating these media in the production of sound-based art can be one way of exposing and exploring these connections. This paper has introduced Click::RAND, an object-based sound installation that utilises a series of relays as its sounding elements. Using a media archaeological approach, the work appropriates the electromechanical relay as a sounding object and, through its creative use as a sound-based installation, connects its use in early electromechanical computers with another obsolete object in the form of a book. As such, Click::RAND creates a media archaeological relationship between the relay and random number sets within the genealogy of computing.

By extending Click::RAND to create a series of works, different perspectives of how the work is experienced can be 
explored. By iterating the basic sound unit through a series of reconfigurable 'sound blocks', the work can be adapted to the space it inhabits. Combining this with different ways to utilise the random number sets a more immersive series of works can be experienced. Developing Click::RAND as a multidimensional set of works continues an ongoing dialogue and interaction between media archaeological artefacts and an audience.

\section{REFERENCES}

[1] S. Zielinski, Deep time in the Media. Toward an Archaeology of Hearing and Seeing by Technical Means., Massachusetts: MIT Press, 2006, p. 3.

[2] RAND, A Million Random Digits with 100,000 Normal Deviates, New York: The Free Press, 1955.

[3] J. Pigott, "Across Fields: Sound, art and technology from an electromechanical perspective," Organised Sound, vol. 22, no. 2, pp. 276-285, 2017.
[4] P. E. Ceruzzi, "Relay Calculators," in Computing Before Computers, Iowa, Iowa State University Press, 1990, pp. 200-222.

[5] L. Landy, Understanding the Art of Sound Organisation, Cambridge, MA: MIT Press, 2007.

[6] P. Vickers, "Sonification and Music, Music and Sonification.," in The Routledge Companion to Sounding Art, London, Routledge, 2016, pp. 135-144.

[7] M. Cobussen, "Introduction," in The Routledge Companion to Sounding Art, London, Routledge, 2016, pp. 95-100.

[8] S. Gresham-Lancaster, "Relationships of sonification to music and sound art," AI \& Society, vol. 27, pp. 207-212, 2012. 